\title{
Silver-Polystyrene (Ag/PS) Nanocomposites Doped with Polyvinyl Alcohol (PVA)-Fabrication and Bactericidal Activity
}

\author{
Anna Krzywicka and Elżbieta Megiel * (1) \\ Faculty of Chemistry, University of Warsaw, Pasteura 1, 02-093 Warsaw, Poland; krzywaa95@gmail.com \\ * Correspondence: emegiel@chem.uw.edu.pl
}

Received: 15 October 2020; Accepted: 8 November 2020; Published: 12 November 2020

\begin{abstract}
In the present work, we report the studies on perfectly homogeneous nanocomposites composed of polystyrene-grafted silver nanoparticles (Ag@PS) as a bioactive fulfilment and a mixture of polystyrene (PS) and polyvinyl alcohol (PVA) as a matrix. The procedure developed by our group of the nanocomposites' preparation consists of three steps: synthesis of narrow-dispersive AgNPs $(5.96 \pm 1.02 \mathrm{~nm})$; grafting of narrowly dispersed polystyrene onto the surface of AgNPs; thermoforming with a mixture of PS/PVA. Kirby-Bauer (K-B) and Dynamic Shake Flask (DSF) assays revealed high antibacterial activity against a series of $\operatorname{Gram}(-)$ and $\operatorname{Gram}(+)$ bacteria strains of the fabricated nanocomposites at low silver content $(0.5 \%)$. We showed that the doping of Ag/PS composites with PVA increases the antibacterial activity of composites. The hydrophilic component in the nanocomposites enables easier water migration inside the polymer matrix, which makes releasing silver nanoparticles and silver ions to the environment facile.
\end{abstract}

Keywords: nanocomposites; silver nanoparticles; polystyrene; nitroxides; antibacterial agents

\section{Introduction}

Nanocomposites (NCs) with silver nanoparticles (AgNPs) as fulfilments and polymers as a matrix are intensively studied due to their useful properties resulting from the unique properties of nanosilver and easy processability of polymers. Among a wide range of applications of such materials, the utilization as antibacterial agents is the one most intensively investigated [1]. Although the biocidal activity of silver has been known for centuries, the current rapid development of nanotechnology gives new opportunities in designing biomedical materials containing this element in the form of AgNPs with well-defined composition and structure. Noteworthily, AgNPs exhibit not only a broad antibacterial activity but also antifungal, antiviral, anti-inflammatory, anti-angiogenic, and anti-cancer activities [2]. Whereas, many reports revealed low/relatively low cytotoxicity of AgNPs towards human cells. However, it must be emphasized that the cytotoxicity of nanoparticles depends on many factors. The most important are size, shape, size distribution, surface charge, and kind of the ligands attached on their surface [3-5]. Thus, the designing of repeated procedures allowing us to obtain AgNPs with precisely defined morphology and structure is crucial for their biomedical applications. Furthermore, the dispersion of AgNPs in an adequately chosen polymer matrix ensures their long-time stability, enables easy processability of such materials, and allows controllable releasing of the nanoparticles from NCs into the environments [6].

There are three main types of nanoparticle-polymer composites (AgNCs) differing in the structure of the nanoparticles used for their preparation and the manner of their combination with the polymer matrix: (1) AgNPs with non-polymer coating dispersed in the polymer matrix; (2) AgNPs attached to the polymer net, and (3) AgNPs with the polymer-modified surface (core-shell nanostructures) dispersed 
in the polymer matrix [6,7]. Only in the last case, homogenous composites with a uniform distribution of nanoparticles in a polymer matrix may be achieved [8]. In the current scientific literature, a layer built of polymer chains covalently grafted onto the nanoparticles' surface is named a polymer brush. Three parameters are crucial for the controlling of AgNPs dispersion in a polymer matrix: (1) grafting density of polymer brush, (2) degree of polymerization of the grafted macromolecules, and (3) degree of polymerization of macromolecules that building the polymer matrix [8]. Additionally, when the structure of matrix polymers and grafted polymers is different, this factor should also be taken into consideration.

In the case of biomedical applications of NCs with AgNPs, the uniform dispersion of fulfilment is crucial because it enables gradual and steady releasing of the antibacterial agent in the form of nanoparticles or silver ions. Noteworthily, the releasing also depends on the polymer matrix affinity to the medium where the NCs are located [9-13].

Great attention is targeted at the AgNCs fabricated from engineering polymers that ensuring their easy processability by typical polymer processing techniques such as injection molding, extrusion, or thermoforming [1].

Polystyrene (PS) is one of the most often commercially used engineering polymers. It is a thermoplastic polymer used in the production of packages, insulation boards, and numerous consumer goods $[14,15]$ as well as medical products such as surgical instruments, dental tools, dressing materials, prosthesis, and many others [16]. For all these applications, uses of PS composites that exhibit biocidal activities, for instance, thanks to the dispersed AgNPs, are highly desirable. However, due to the hydrophobic character of this polymer, the preparation of stable and homogeneous composites requires a proper modification of the nanoparticles' surface. There are a few reports on the modification of AgNPs with hydrophobic molecules such as oleylamine [17] and polystyrene [18,19] via their physical adsorption onto the nanoparticles' surface. Although hydrophobic modification of AgNPs' surface undoubtedly facilitates their dispersion in the hydrophobic matrix, this loosely adsorbed layer cannot efficiently protect the nanoparticles against aggregation during the thermal processing of such materials.

Grafting of polymers onto the surface of nanoparticles means anchoring via covalent bonds (also called polymer brush formation), and it enables the preparation of nanohybrids that are miscible in the polymer matrix and also resistant against aggregation [8].

Recently, our group proposed a novel approach for the preparation of perfectly homogeneous Ag/PS nanocomposites using high-density polystyrene grafted silver nanoparticles. The developed procedure is based on utilizing nitroxide-mediated polymerization (NMRP) and relies on the coupling of growing polystyrene chains with nitroxide-coated silver nanoparticles. These fabricated nanohybrids are readily dispersible in the polystyrene matrix. The prepared composites Ag/PS are thermoforming and exhibit significant antibacterial activity against model Gram(-) (Pseudomonas aeruginosa) and model Gram(+) (Staphylococcus aureus) pathogenic bacteria. Nevertheless, the antibacterial effect is strong when silver content is relatively high $\geq 2 \%(w / w)$ [20]. Thus, it would be worth to find a means of enabling of decreasing silver content and maintain the same bioactivity. We hypothesized that the introduction into the polystyrene matrix of a hydrophilic polymer admixture would allow for enabling easier silver ion release thanks to more effective water migration inside of the material. Therefore, an excellent antibacterial activity could be achieved for lower silver content. For this purpose, we decided to use poly(vinyl alcohol)_PVA as a hydrophilic admixture for the PS matrix. The PVA is widely used for the fabrication of biomaterials as biocompatible, biodegradable, water-soluble, and at the same time, non-expensive polymers [21,22]. Therefore, the bioactive nanocomposites with PVA as a component can find plenty of interesting applications, among others, in medicine, cosmetics, pharmaceuticals, and the packaging industry.

Very recently, Abdallah and co-workers proved that the nanocomposites with AgNPs as fulfilment and PVA as a matrix exhibit high antibacterial activity against a series of Multi-Drug Resistance bacteria strains [23]. 
In this work, we present research that reveals the influence of hydrophilic admixture in nanocomposites' matrix on their antibacterial activity. We developed the procedure allowing us to obtain perfectly homogeneous PS/PVA/Ag nanocomposites containing silver nanoparticles grafted with polystyrene.

Notably, the developed method allows for the preparation of the nanocomposites on a scale of tens of grammes. The developed modification of AgNPs gives them perfect miscibility with the polymer matrix and at the same time high thermal stability. Due to the connection of polystyrene chains via the nitroxide linker with the silver surface, the polymer shell is covalently attached to the nanoparticle. Whereas, the hydrophilic admixture (PVA) allows for gaining efficient antibacterial activity at low silver content.

The prepared nanocomposites with silver content $2 \%, 1 \%$, and $0.5 \%$ were subjected to the comprehensive antibacterial assays towards four strains of Gram(-) type and three strains of Gram(+) using two types of antibacterial assays: the Kirby-Bauer disc susceptibility test and the Dynamic Shake Flask method. The composites fabricated, according to the reported procedure, exhibit high antibacterial activity at silver content as low as $0.5 \%$.

The detailed physicochemical characterizations of the fabricated nanomaterials were carried out using UV-vis spectrophotometry, Size-exclusion chromatography (SEC), Transmission Electron Microscopy (TEM), and Thermogravimetric analysis (TGA).

\section{Materials and Methods}

Styrene (Sigma Aldrich, St. Louis, MO, USA, puriss $\geq 99 \%$ ) was dried over $\mathrm{MgSO}_{4}$ and passed through a basic aluminum oxide column before use (Merck, Kenilworth, NJ, USA, $0.06 \mathrm{~mm}$ ). Benzoyl peroxide (BPO) was recrystallized twice from the chloroform-methanol mixture and dried in a desiccator; $\mathrm{NaBH}_{4}, \mathrm{AgNO}_{3}, 4$-hydroxy-TEMPO (TEMPOL), PVA $\left(\mathrm{M}_{\mathrm{w}}=27 \mathrm{kDa}\right)$ with a high degree of hydrolysis (98\%), and all solvents were purchased from Sigma-Aldrich (St. Louis, MO, USA, puriss $\geq 97 \%$ ) and used as received.

Bis (N-oxy-2,2,6,6-tetramethylpiperidyl)-4,5-dithiooctanoate (DiSS) was synthesized according to the procedure reported in our earlier work [24]. Milli-Q ultrapure water (resistivity $18.2 \mathrm{M} \Omega \mathrm{cm}^{-1}$, Millipore-Merk, Boston, MA, USA) was used throughout the experiments.

\subsection{Microbial Strains}

Gram(-): Escherichia coli-source ATCC 25922, Salmonella enterica sv Typhimurium-St11 strain, Yersinia enterocolitica-Ye9 strain; Pseudomonas aeruginosa-PAO1 strain; Campylobacter jejuni-Cj 22 source of the last three strains-Institute of Microbiology collection (University of Warsaw, Faculty of Biology, Warsaw, Poland).

Gram(+): Staphylococcus aureus-source ATCC 43300; Bacillus cereus-source ATCC 10876; Listeria monocytogenes-EGD strain, source-Institute of Microbiology collection (University of Warsaw, Faculty of Biology, Warsaw, Poland).

\subsection{Techniques}

\subsubsection{Transmission Electron Microscopy (TEM)}

Transmission Electron Microscopy (TEM) analyses were performed using the JEM 1400 JEOL Co. microscope (JEOL, Tokyo, Japan) at $120 \mathrm{kV}$ acceleration voltage.

The solution of the sample (with concentration $1 \mathrm{mg} / \mathrm{mL}$ ) was cast onto a carbon-coated copper microgrid (200 mesh) and air-dried. For the preparation of the solutions of nanocomposites and AgNPs, $\mathrm{THF}$, and acetone were used, respectively. 


\subsubsection{UV-vis Spectrophotometry}

UV-vis spectra were recorded using a Cary 50 Conc UV/Vis spectrophotometer (Varian, Palo Alto, CA, USA). The spectra were recorded in N, N-dimethylformamide (DMF) solutions.

\subsubsection{Thermogravimetric Analyses (TGA)}

Thermogravimetric (TG) measurements were performed using a TA Instruments DSC Q20 apparatus (TA instruments, New Castle, DE, USA) with thermobalance (precision $\pm 0.4 \%$; minimal mass $0.02 \mathrm{mg}$ ) under an $\mathrm{N}_{2}$ atmosphere with scanning rate $10 \mathrm{~K} \mathrm{~min}^{-1}$.

\subsubsection{Size-Exclusion Chromatography (SEC)}

The Size-Exclusion Chromatography (SEC) analyses were carried out using Waters Alliance 2695 liquid chromatograph equipped with a RID detector (Waters 2414 RI, Milford, MA, USA).

The separations of PSs were performed in tetrahydrofuran (THF) on three columns placed in series: Waters Styragel HR1, HR2, HR4 $7.8 \times 300$ mm (Waters, Milford, MA, USA); The columns were thermostated at $35^{\circ} \mathrm{C}$. HPLC grade THF (Sigma-Aldrich, St. Louis, MO, USA) was used at a flow rate of $0.8 \mathrm{~mL} \mathrm{~min}^{-1}$. The concentration of samples prepared in eluents was $1 \mathrm{mg} \mathrm{mL}^{-1}, 20 \mu \mathrm{L}$ injections were applied. PS standards were used for calibration (Shodex, Showa Denko K. K., Tokyo, Japan) in the range $1.31 \times 10^{3}$ to $3.64 \times 10^{6} \mathrm{Da}$.

\subsubsection{Preparation of Polystyrene Grafted Silver Nanoparticles (Ag@PS)}

The first step was the preparation of nitroxide-coated silver nanoparticles (N-AgNPs) that can be united with polystyrene macroradicals thanks to the unpaired electrons localized in nitroxide moieties.

Recently, we developed an efficient protocol for the synthesis of N-AgNPs on a scale of tens of micrograms. In this paper, we report a modification of this method, allowing us to fabricate the nanoparticles on a scale of hundreds of milligrams/grams using a lab set composed of the jacketed reactor (with capacity $0.5 \mathrm{~L}$ ) equipped with a mechanical stirrer connected with the thermostat controlling temperature of the heat/cooling medium in the jacket with an accuracy of $\pm 0.5^{\circ} \mathrm{C}$ in the range $-20-130^{\circ} \mathrm{C}$. Figure S1 in the Supplementary Materials shows the photograph of the designed lab set that was used in the preparation of N-AgNPS. The developed protocol is as follows: $677.7 \mathrm{mg}$ of DiSS (1.321 mmol) was dissolved in $300 \mathrm{~mL}$ of DMF in the reactor, and the mixture was bubbled with argon (for $20 \mathrm{~min}$ ) and stirred using a mechanical stirrer (1600 rpm); the temperature of the cooling medium was set at $-20^{\circ} \mathrm{C}$. When the mixture achieved the set temperature, $1.115 \mathrm{~mL}$ of $\mathrm{AgNO}_{3}$ solution with concentration $1 \mathrm{M}(1.115 \mathrm{mmol})$ was injected and after 5 min a solution of $\mathrm{NaBH}_{4}$ (75.3 mg, $2 \mathrm{mmol}$ ) in DMF (120 mL) was added using a peristaltic pump (Thermo Fisher Scientific) with a velocity of pumping $1.5 \mathrm{~mL} / \mathrm{min}$. During the addition of the solution of $\mathrm{NaBH}_{4}$ stirring of the mixture was accelerated to $3000 \mathrm{rpm}$. After addition of the reducing agent solution, the mixture became a chocolate-brown color; the stirring was continued with a velocity of $1600 \mathrm{rpm}$ for the next $2 \mathrm{~h}$. Afterwards, the post-reaction mixture was mixed with water (ultra-pure $500 \mathrm{~mL}$ ), and a pinch of $\mathrm{NaCl}$ was added to precipitate the fabricated nanoparticles. The obtained suspension was sonicated ( $2 \mathrm{~min})$, centrifuged (10,000 rpm, $10 \mathrm{~min})$, the supernatant was discarded, and the precipitate was washed several times profusely with water (ultra-pure). The obtained solid was dried in a vacuum oven at $50^{\circ} \mathrm{C}$ for $24 \mathrm{~h}$. Thin-layer chromatography (TLC) confirmed the absence of impurities and not-attached ligands in the solution of the prepared N-AgNPs. Finally, the grey, solid product was obtained (160 mg, yield $75 \%$, the yield was recalculated on silver content that was taken from TGA).

The fabricated N-AgNPs were used in the preparation of Ag@PSs via coupling with polystyrene macroradicals during nitroxide-mediated polymerization of styrene. The employed procedure was developed very recently by our group [20]. In this paper, we report the succeeded rescaling of this protocol, allowing us to fabricate nanocomposites in gram quantities and their blends with poly(vinyl alcohol) (PVA). 
Styrene (45 mL, $392 \mathrm{mmol})$, TEMPOL $(0.409 \mathrm{~g}, 2.40 \mathrm{mmol})$ and BPO (0.194 g, $0.80 \mathrm{mmol})$ were inserted to the Schlenk flask sealed with a silicone rubber septum. The applied molar ratio of reactants St:TEMPOL: BPO was 490:3:1 that allowed for obtaining polystyrene (PS) with a maximum average molecular mass equal to $50.96 \mathrm{kDa}$ (when the monomer conversion reached $100 \%$ ).

The polymerization mixture was degassed using the four freeze-pump-thaw cycles procedure; next pure and dry argon was introduced to the flask, and the flask was immersed in a thermostatically controlled oil bath, set at $120{ }^{\circ} \mathrm{C}$ and placed on a magnetic stirrer. After $24 \mathrm{~h}$ from the mixture $5 \mathrm{~mL}$ of sample was taken and $20 \mathrm{~mL}$ of an acetone solution containing $140 \mathrm{mg}$ N-AgNPs was injected into polymerization mixture using a syringe (in the counter flow of argon). Notably, the acetone solution of $\mathrm{N}$-AgNPs was degassed for $30 \mathrm{~min}$ by dry argon bubbling before the injection.

The polymerization of styrene with $\mathrm{N}$-AgNPs was continued for $5 \mathrm{~h}$. After this time, the reaction was stopped by cooling the flask and opening to contact the mixture with air. The post-polymerization mixture was viscous, chocolate-brown, and homogeneous, and any signs of nanoparticles aggregation were not observed.

Silver nanoparticles grafted with polystyrene (Ag@PS) were isolated from the mixture with free polystyrene chains (not attached to the surface) via the precipitation process with n-hexane as the precipitant and THF as a solvent. First, the mixture was dissolved in THF, 5 -times excess of $\mathrm{n}$-hexane was added, and the suspension obtained in this way was centrifuged $(10,000 \mathrm{rpm}, 10 \mathrm{~min})$. The process of purification was repeated until turbidity was not observed after the addition of methanol to the supernatant. The brown-solid product was dried in a vacuum oven for $24 \mathrm{~h}$ at $60{ }^{\circ} \mathrm{C}$ (a final mass of the product was $520 \mathrm{mg}$ ).

\subsubsection{Preparation of Nanocomposites PS/PVA/Ag@PS and Their Thermoforming into pastilles}

The composites were fabricated using a home-made pellet press die set built of Teflon two disks placed in Teflon cylinder with a proper size channel. Two types of pastilles were prepared: with a diameter of $0.5 \mathrm{~cm}$ and mass ca. $30 \mathrm{mg}$ and bigger with diameter $1.5 \mathrm{~cm}$ and mass ca. $70 \mathrm{mg}$. The first of them were used in the Kirby-Bauer disc susceptibility test and the second ones in Dynamic Shake Flask tests.

The polystyrene (PS) used for the NCs preparation was isolated from the sample taken during synthesis Ag@PS before the addition of N-AgNPs (see above). SEC analysis performed for this PS sample displayed an average number molecular weight $\mathrm{M}_{n}=10.8 \mathrm{kDa}$, an average molecular weight at the maximum of peak $11.39 \mathrm{kDa}$, and the polydispersity index equals 1.13 .

The appropriate concentration of nanosilver in the pastilles was achieved by mixing Ag@PS with a concentration of Ag 18\% (determined from TGA) and proper amounts of PS and PVA to gain, at the same time, $5 \%$ of PVA.

The general procedure of the pastilles preparing was the following: a mixture of PS, Ag@PS and PVA in proper proportions and mass $40 \mathrm{mg}$ or $85 \mathrm{mg}$ was ground using a bead mill for $10 \mathrm{~min}$. The prepared powder was placed between two disks inside a channel in the press die set. Afterwards, this mixture was pressed using a laboratory press. The fabricated pastilles were transferred onto a Petri dish, placed on the thermal plate of a magnetic stirrer, and heated at $150{ }^{\circ} \mathrm{C}$. As a result, the pastilles became vitreous, and after cooling them to room temperature, they could be taken out.

\subsubsection{Antibacterial Assays of Ag/PS/PVA}

Two types of assay were used to assess the antibacterial activity of the fabricated nanocomposites. Both of them show the ability to release antibacterial agents from solid materials. They differ in their mean of contact of the materials with bacterial strains.

Kirby-Bauer Disc Diffusion Susceptibility Test

Kirby-Bauer disc diffusion susceptibility tests were performed according to EUCAST recommendations [25]. The bacterial cultures were diluted to an optical density of 0.5 McFarland 
standard and swabbed on the surface of Mueller-Hinton agar plates. The pastilles made of composites (Ag/PS/PVA) with the mass $36 \pm 5 \mathrm{mg}$ were placed in the agar, and the plates were incubated at $37^{\circ} \mathrm{C}$ for $24 \mathrm{~h}$. The zones of inhibition (ZI) were measured as the average from three separate experiments.

\section{Dynamic Shake Flask Method (DSFM)}

DSF assays were performed in the phosphate buffer in the absence of the nutrient, thus under no bacteria growing conditions.

The flasks containing the phosphate buffer $(0.3 \mathrm{M}, \mathrm{pH}=7.2)$ was inoculated with $10^{5} \mathrm{CFU} \mathrm{mL}^{-1}$ (colony-forming units per millilitre) bacteria. To each flask, the tablets made of composite (Ag/PS/PVA) with the mass $76 \pm 8 \mathrm{mg}$ were placed. The pastilles were not sterilized before contact with the bacteria. The flasks were shaken at $200 \mathrm{rpm}$ for $24 \mathrm{~h}$ at $37^{\circ} \mathrm{C}$. Furthermore, analogous cultures were carried out with the tablet made of polystyrene mixed with PVA $5 \%(w / w)$ with the mass $75 \pm 5 \mathrm{mg}$ (not sterilized) as a control test. After appropriated periods of contact time, the bacterial concentrations of the microbial suspension were determined by measuring optical density (O.D.) at wavelength $600 \mathrm{~nm}$.

\section{Results and Discussion}

The nanocomposites of polystyrene grafted silver nanoparticles (Ag@PS) with a mixture of polystyrene (PS) and polyvinyl alcohol (PVA) were fabricated. The developed procedure allows for preparing perfectly homogeneous nanomaterials exhibiting antibacterial properties at a very low content of nanosilver $(0.5 \% w / w)$.

Figure 1 displays TEM images of nitroxide-coated silver nanoparticles (N-AgNPs), the nanohybrids Ag@PS prepared from them, and the fabricated nanocomposites (Ag/PS/PVA).

a) N-AgNPs

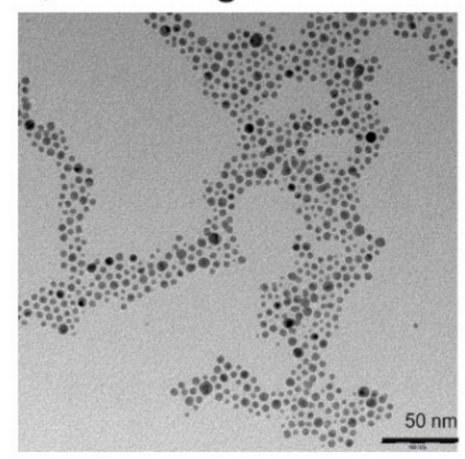

b) Ag@PS

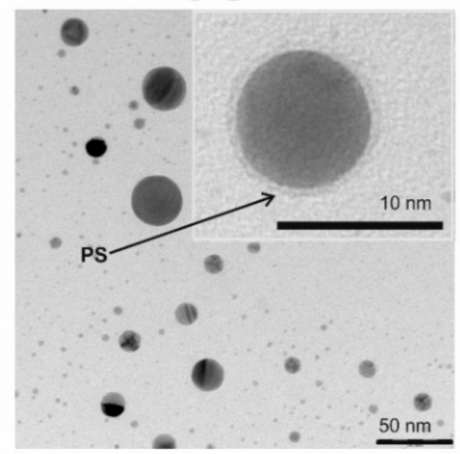

c) $\mathrm{Ag} / \mathrm{PS} / \mathrm{PVA}$

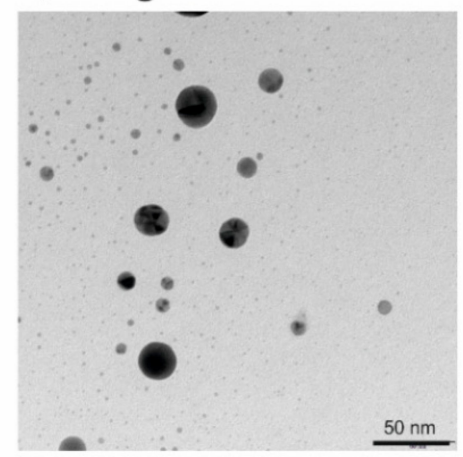

Figure 1. TEM images of the synthesized nanomaterials: nitroxide-coated silver nanoparticles (a), polystyrene grafted silver nanoparticles $(\mathbf{b})$ and nanocomposites of polystyrene grafted silver nanoparticles with polystyrene doped with polyvinyl alcohol (95:5 w/w PS:PVA) (c).

As can be seen in Figure 1a, the nitroxide-coated silver nanoparticles (N-AgNPs) are narrowly dispersed in terms of their size. Further, they have the ability to self-assemble, which is most probably a consequence of interactions between nitroxide radicals and silver. Such interactions cause accumulation of the nanoparticles in partially ordered structures but importantly do not lead to the aggregation. Recently our group reported the existence of the interactions of nitroxide radicals with silver and gold nanoparticles/surfaces [24,26-29].

The presence of nitroxide radicals covalently connected with the silver surface in N-AgNPs allows us to connect polystyrene-growing macroradicals during the polymerization and obtain in this way polystyrene grafted silver nanoparticles (Ag@PS). During the polymerization, a fraction of nanoparticles increases in size, as can be seen in Figure 1b.

The inset in Figure $1 \mathrm{~b}$ displays the TEM image of the single silver nanoparticle (magnification with the scale bar $10 \mathrm{~nm}$ ) where the surrounding polymer shell onto the nanoparticle is visible. 
Figure 2 displays the histograms drawn on the base of TEM images shown in Figure 1. As can be seen in Figure 2 size distribution of N-AgNPs is very narrow, and during the polymerization of styrene with $N$-AgNPs part of nanoparticles increases their size even to above $35 \mathrm{~nm}$, but $84 \%$ of the synthesized nanohybrids (Ag@PS) have a diameter below $10 \mathrm{~nm}$. The size distribution is very similar in the nanocomposites fabricated using Ag@PS as fulfilment and the mixture of PS and PVA as a matrix.

TEM studies revealed that the nanocomposites with homogeneously dispersed, non-aggregated spherical silver nanoparticles with the diameter mostly below $10 \mathrm{~nm}$ (see Figure 2) were prepared.

Due to the strong localized surface plasmon resonance (LSPR) of silver nanoparticles, the UV-vis spectrophotometry is a very convenient tool to analyze their dispersity in a polymer matrix. The position of LSPR band/bands in the absorption spectrum and its full width at half maximum (FWHM) depend not only on the shape, size, and dispersity of AgNPs, but also on the refractive index of the environment (solvent or solid matrix) [17,30,31].

a)

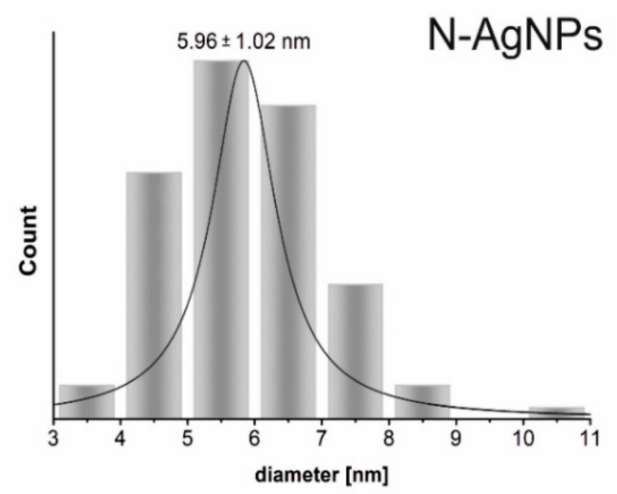

b)

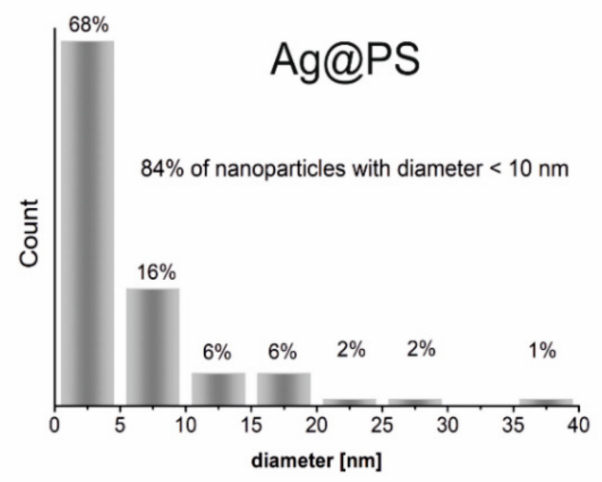

c)

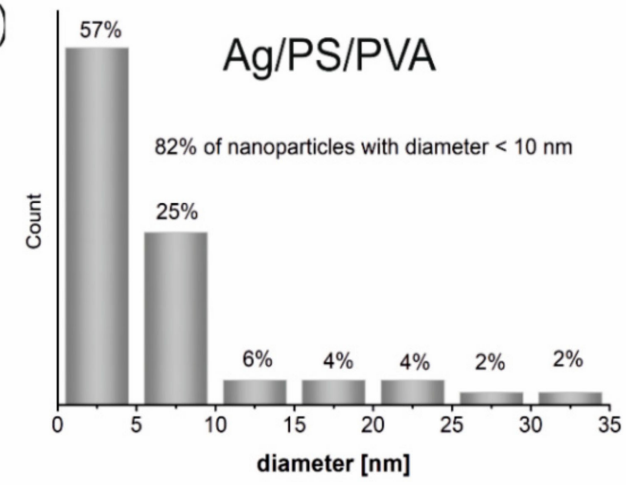

Figure 2. The histograms of the size distribution of silver nanoparticles in the fabricated nanomaterials: nitroxide-coated silver nanoparticles (a), polystyrene grafted silver nanoparticles (b) and nanocomposites of polystyrene grafted silver nanoparticles with polystyrene doped with polyvinyl alcohol (95:5 $w / w$ PS:PVA) (c). The histograms have been drawn on the base of TEM images shown in Figure 1.

Figure 3 shows the absorption spectra of the prepared silver nanoparticles; polystyrene-grafted nanohybrids and nanocomposites in DMF solutions. The LSPR band in N-AgNPs spectrum with a maximum at $416 \mathrm{~nm}$ is narrow and symmetric and confirms the narrow dispersity of the nanoparticles. The position of the maximum absorption correlates well with the average size of metal cores ca. $6 \mathrm{~nm}$ [28]. For Ag@PS, the LSPR band is red-shifted to $427 \mathrm{~nm}$ compared with the band in N-AgNPs spectrum. This effect can be explained by changing the refractive index of the protecting layer attached to the surface of the nanoparticles. The maximum of the absorption band for the nanocomposite Ag/PS/PVA is significantly more red-shifted to $442 \mathrm{~nm}$, and also the width of LSPR has increased. It indicates that during their thermal processing, widening of the size dispersity of silver nanoparticles occurs. It is consistent with the results from TEM analyses (see Figures 1 and 2). Notably, the changes in 
size dispersity are not significant (ca. $80 \%$ of nanoparticles retain the same size). In the spectrum of Ag/PS/PVA, two bands characteristic for polystyrene in the UV range at 220 and $260 \mathrm{~nm}$ are visible. The absorption bands of PVA in UV-vis spectra are not visible, due to its low concentration in the nanocomposites $(5 \% w / w)$. We recorded the spectrum of PVA in the same solvent at the same weight concentration, and any bands were not visible.

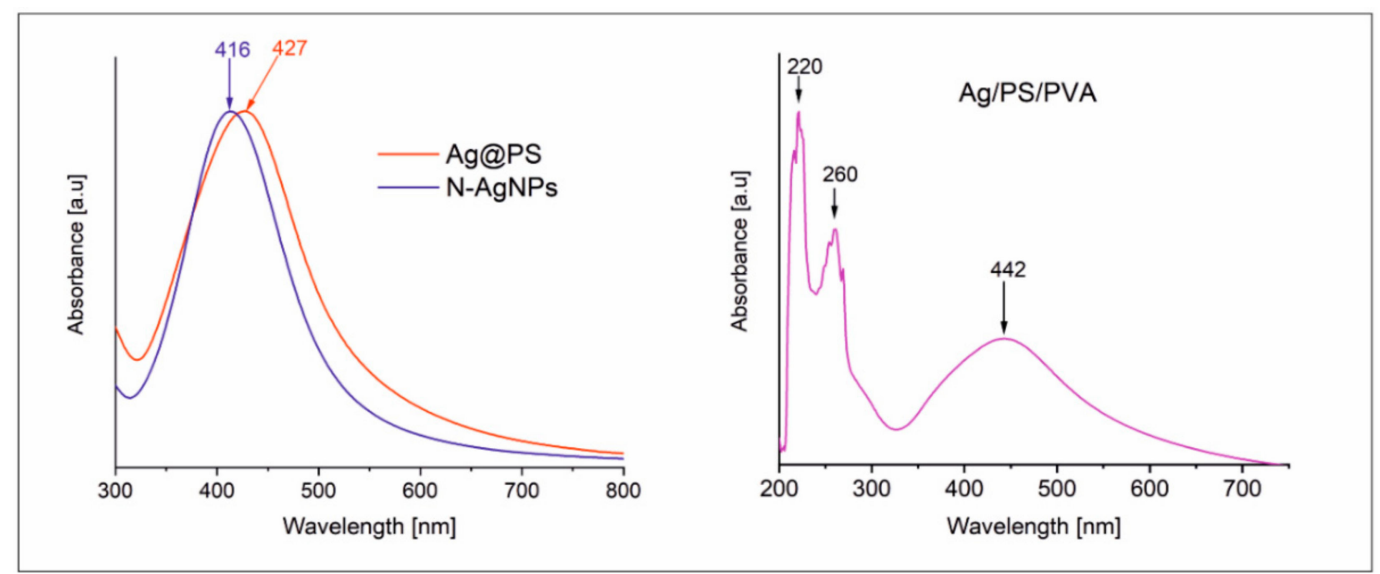

Figure 3. UV-vis absorption spectra recorded for the fabricated nanomaterials (left) Ag@PS, N-AgNPs and (right) $\mathrm{Ag} / \mathrm{PS} / \mathrm{PVA}$ in DMF solutions at $298 \mathrm{~K}$.

The developed procedure for the preparation of Ag@PS is based on the employment of the nitroxide mediated polymerization (NMRP) [32], allowing us to prepare narrowly dispersed polymers with a well-defined molecular weight. Recently, our group developed the method for the grafting of polymer chains on the surface of nanoparticles using NMRP $[20,28,29,33]$. In this method, nitroxide-coated nanoparticles are injected to the polymerization system controlled by a nitroxide radical. During the polymerization, the growing macroradicals are captured by the radicals attached on the nanoparticles' surface. In this way, nanohybrid structures with covalently attached polymer chains are obtained (in the case of silver nanoparticles denoted as Ag@PS). Besides the nanohybrids, in the polymerization system, free ones not attached to the nanoparticles polymer chains are prepared with well-defined molecular weights.

Now we use Ag@PS nanohybrids for the fabrication of the Ag/PS/PVA nanocomposites. The polystyrene applied for this purpose (PSF) was separated from the same polymerization system as Ag@PS. The size exclusion chromatography (SEC) was used to determine average molecular weights and dispersity of PSF. Table 1 displays the results of the SEC analysis of PSF. The elugram and molecular weights distribution are presented in the Supplementary Information (Figures S2 and S3, respectively).

Table 1. The average molecular weights determined from SEC analysis for polystyrene that was used for the nanocomposites' fabrication.

\begin{tabular}{cccccc}
\hline Sample & $\mathbf{M}_{\mathbf{n}}[\mathrm{Da}]$ & $\mathbf{M}_{\mathbf{w}}[\mathrm{Da}]$ & $\mathbf{M}_{\mathbf{p}}[\mathrm{Da}]$ & $\mathbf{M}_{\mathbf{z}}[\mathrm{Da}]$ & $\mathbf{P D I}$ \\
\hline PSF & 10,350 & 11,700 & 11,390 & 13,180 & 1.13 \\
\hline
\end{tabular}

Due to the application of the nitroxide radical (TEMPOL) as a mediator, polystyrene formed in the polymerization system (PSF) is narrowly dispersed (polydispersity index PDI $=1.13$ ). In our earlier paper, we proved that the molecular weight of polymers connected with nanoparticles and free in the system is similar. This is so because, during the polymerization, an exchange between attached and free polymer chains is possible [20]. As a consequence, Ag@PS is perfectly miscible in the polymer matrix (PSF) used for the nanocomposites' (Ag/PS/PVA) preparation. PVA with an averaged molecular weight $M_{W}=27 \mathrm{kDa}$ was used for the nanocomposites' preparation. Notably, the composites with the 
concentration of PVA above 5\% (w/w) were becoming non-homogeneous during their thermoforming. Therefore, for all studies, we decided to use only the nanocomposites with 5\% PVA.

Thermogravimetric analyses (TGA) were conducted to evaluate the stability of the fabricated nanomaterials. Figure 4 shows TGA curves in the range $20-600{ }^{\circ} \mathrm{C}$ and the corresponding first derivatives (DTA).

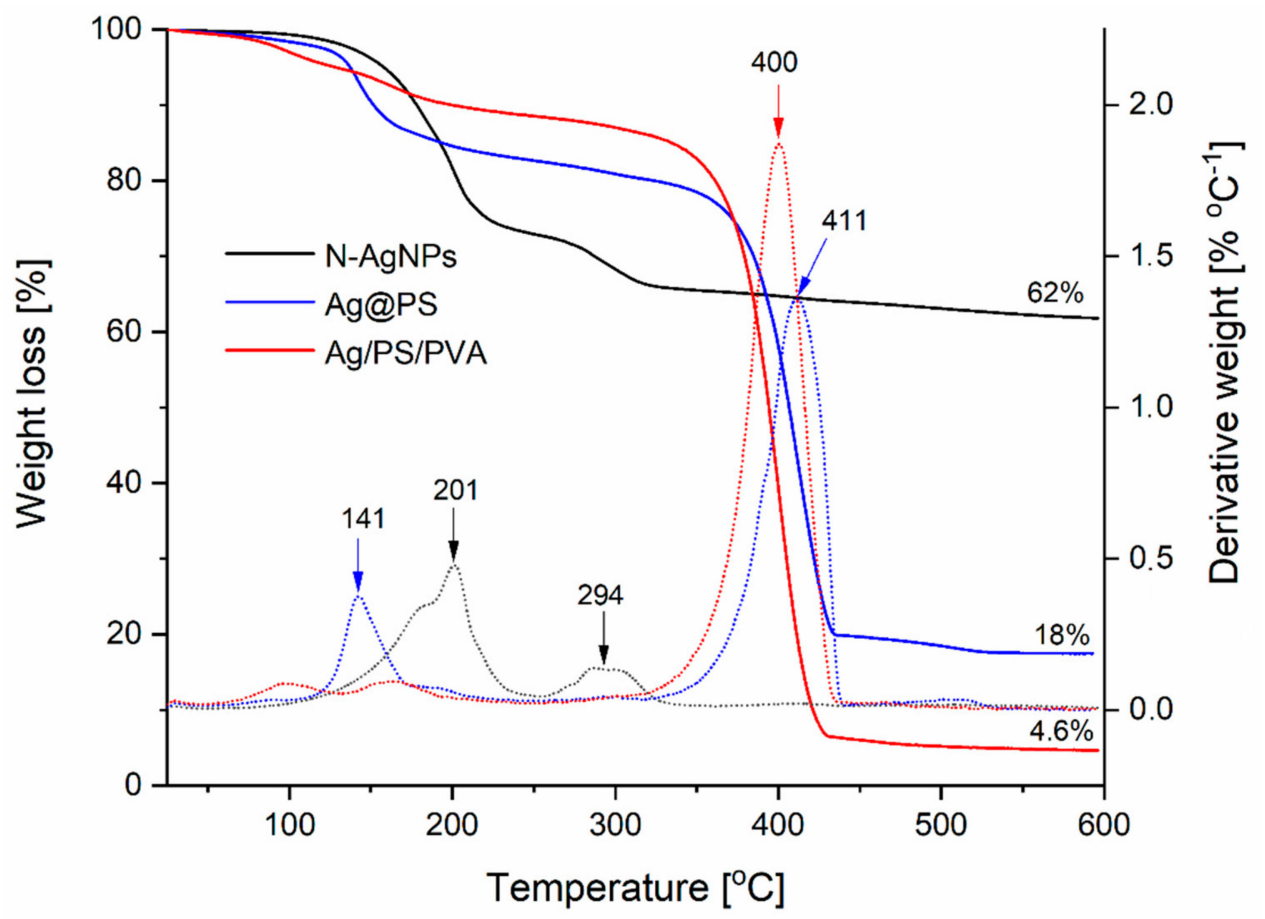

Figure 4. Thermogravimetric analysis (TGA) curves (solid lines) and corresponding first-derivatives of TGA with respect to the temperature (dotted lines with the same colour) of nitroxide-coated silver nanoparticles (N-AgNPs), nanohybrids with polystyrene (Ag@PS), their nanocomposites with polystyrene, and polyvinyl alcohol (Ag/PS/PVA). The given above the TGA curves the final percentage of start mass corresponds to silver content in the fabricated materials.

As can be seen, the decomposition of nitroxide-coated nanoparticles, under the conditions of analysis, is completed below $300{ }^{\circ} \mathrm{C}$ and occurs in two steps with the maximum weight loss at $201^{\circ} \mathrm{C}$ and $294^{\circ} \mathrm{C}$ (see the first derivative curve of corresponding TGA curve). When the polystyrene chains are attached to the surface of the nanoparticles via nitroxide radicals, a significant increase of thermal stability is observed (see TGA curve for Ag@PS), and the decomposition is completed above $450{ }^{\circ} \mathrm{C}$. However, the first step of the decomposition runs at a lower temperature and maximum is located at $141^{\circ} \mathrm{C}$; this step corresponds to only $15 \%$ of weight loss. Most likely, part of the nitroxide radicals covering the nanoparticles' surface remained not connected with polystyrene chains, and their decomposition runs at a lower temperature.

In the case of nanocomposites of Ag@PS with a mixture of PS and PVA (95\%: 5\%, w/w), the main decomposition step occurs above $300{ }^{\circ} \mathrm{C}$ and corresponds to $80 \%$ of total weight loss. These results confirm that the fabricated nanocomposites can be processed by thermoforming at the temperatures below $200{ }^{\circ} \mathrm{C}$ without decomposition.

The final percentage of the start mass obtained from TGA corresponds to a content of silver in the fabricated materials, and it is $62 \%$ for nitroxide-coated nanoparticles and $18 \%$ in the nanohybrids (Ag@PS) prepared from them.

As was written earlier, the developed procedure allows for obtaining the nanocomposites with different silver contents depending on the ratio Ag@PS and polymer matrix (PS/PVA). In Figure 4, the exemplified TGA curve is present for nanocomposite Ag/PS/PVA with silver content 4.6\%. 
Antibacterial activity of the fabricated nanocomposites was evaluated using two assays, which are commonly used for solid materials, namely the Kirby-Bauer (K-B) disc susceptibility test and the Dynamic Shake Flask (DSF) method. Because of the potential applications of the prepared materials as packaging materials, we decided to exam series of pathogenic bacteria that may be present in food and water. There are Salmonella typhimurium, Escherichia coli, Yersinia enterocolitica, Campylobacter jejuni, Bacillus cereus, and Listeria monocytogenes. Furthermore, antibacterial activity against usually antibiotic-resistant bacterial strains such as Pseudomonas aeruginosa and Staphylococcus aureus was tested.

As it turned out, all fabricated nanocomposites exhibit high antibacterial activity against all studied pathogenic strains, both $\operatorname{Gram}(-)$, and $\operatorname{Gram}(+)$.

Figure 5 shows photographic images of the results of K-B assays for selected bacterial strains. As can be seen the inhibition zones (IZs), it means the area without bacterial growth is distinctly visible for all materials, also for those with the lowest studied silver content, namely $0.5 \%$.

a)

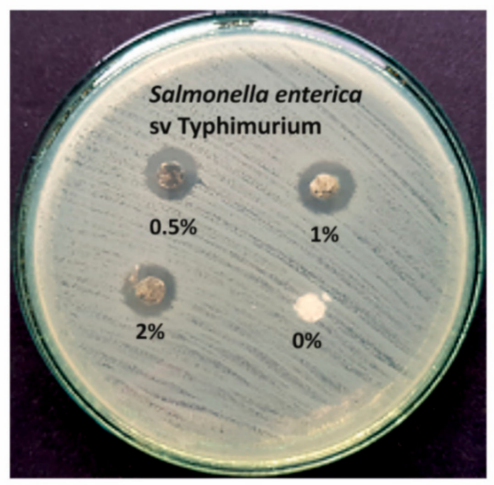

c)

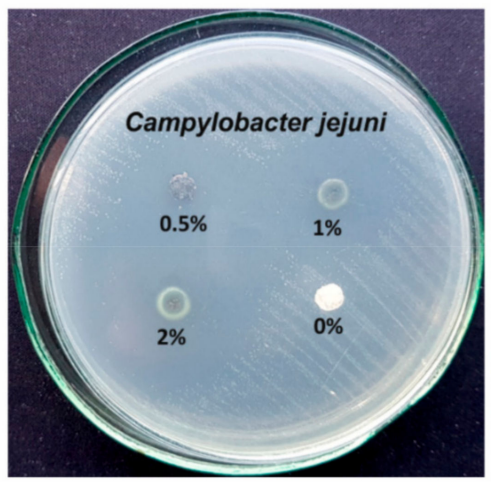

e)

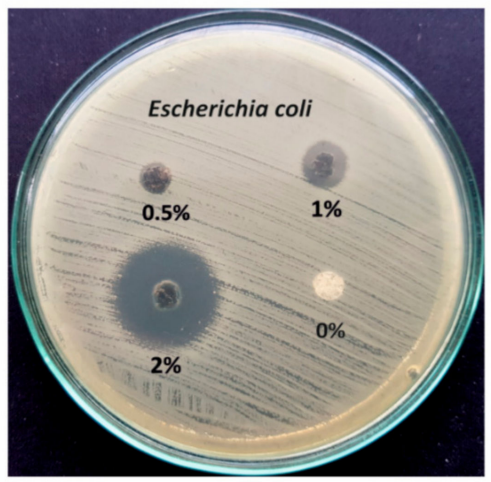

b)

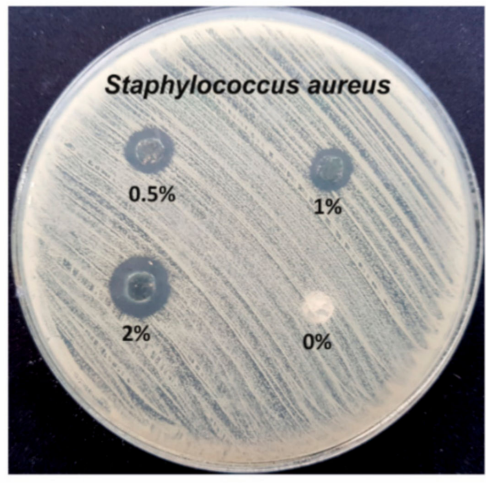

d)

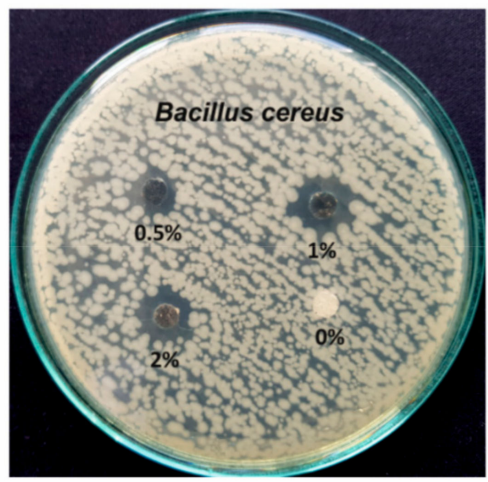

f)

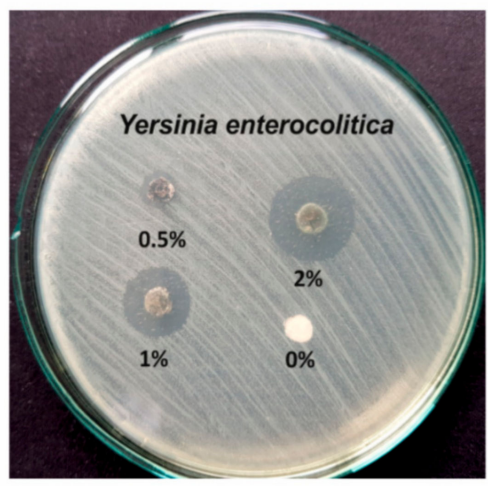

Figure 5. Photograph images of Kirby-Bauer susceptibility test results after $24 \mathrm{~h}$ of incubation at $37^{\circ} \mathrm{C}$ : for Gram(-) strains $(\mathbf{a}, \mathbf{c}, \mathbf{e})$ and $\operatorname{Gram}(+)$ strains $(\mathbf{b}, \mathbf{d}, \mathbf{f})$. The sample composed of the mixture PS with PVA 95:5 $(w / w)$ was denoted as $0 \%$ and used in all tests as a control sample. 
The IZs determined from the performed K-B assays for all studied nanocomposites against all studied bacterial strains are presented in Table 2. As can be seen, IZs are in the range 7-34 $\mathrm{mm}$, it means that the antibacterial activity of the fabricated nanocomposites is good or very good. Our results proved that the introducing of PVA as a hydrophilic component significantly increased antibacterial activity of AgNPs dispersed in a hydrophobic polymer matrix. IZ determined against Pseudomonas aeruginosa, and Staphylococcus aureus determined for the composite PS/Ag@PS without PVA is $6 \mathrm{~mm}$ and $4 \mathrm{~mm}$ at $1 \%$ of nanosilver. Whereas in the case of reported here Ag/PS/PVA, these IZs are equal to $11 \mathrm{~mm}$, so are almost two times higher. The nanocomposites not containing PVA, when nanosilver concentration is below $1 \%$, do not exhibit antibacterial activity. Thus, the introduction of a small amount of a hydrophilic polymer to the hydrophobic matrix dramatically increased the bioactivity of the composite. Most likely, it facilitates water migration inside of the polymer matrix, and this leads to the easier releasing of silver nanoparticles and silver ions.

Table 2. Results of Kirby-Bauer assay for Ag/PS/PVA composites differing nanosilver content (5\% $w / w$ of PVA).

\begin{tabular}{cccc}
\hline \multicolumn{4}{c}{ Inhibition Zones IZ [mm] } \\
\hline \multicolumn{4}{c}{ Gram(-) } \\
\hline \multicolumn{4}{c}{ Content of Nanosilver [\% w/w] } \\
\hline 2 & $\mathbf{1}$ & $\mathbf{0 . 5}$ \\
\hline Salmonella typhimurium & 12 & 11 & 11 \\
Escherichia coli & 22 & 11 & 7 \\
Yersinia enterocolitica & 14 & 17 & 8.5 \\
Pseudomonas aeruginosa & 13 & 11 & 7 \\
Campylobacter jejuni & 34 & 21 & 17 \\
\hline & Gram(+) & & 10 \\
\hline Staphylococcus aureus & 12 & 11 & 10 \\
Bacillus cereus & 11 & 11 & 10 \\
Listeria monocytogenes & 10 & 11 & \\
\hline
\end{tabular}

The determined IZ values for Gram(-) bacterial strains are higher for all the studied nanocomposites in comparison with these determined for $\operatorname{Gram}(+)$. It can be explained by differences in the structure of their cell membranes that cause higher susceptibility of Gram(-) strains [34].

The highest susceptibility, among those studied, exhibits Campylobacter jejuni, the IZ value for the nanocomposite with silver content $0.5 \%$ is as high as $17 \mathrm{~mm}$. It is worth mentioning that Campylobacter is a foodborne pathogen which is responsible for many bacterial diarrheal diseases. At the same time, antibiotic resistance of these strains is observed recently more and more frequently [35]. Thus, all the more, the fabricated nanocomposites have a strong application potential, among others, as food packaging materials.

The Dynamic Shake Flask (DSF) method was also used to evaluate the antibacterial activity of the prepared nanomaterials. This method is commonly used in the case of polymer composites and textiles. We chose three bacterial strains: Escherichia coli as Gram(-) representative, Staphylococcus aureus as Gram(+) representative, and Yersinia enterocolitica-a pathogen often present in food products and water causing acute infections and disease [36]. It is noteworthy that this bacterial strain can withstand low temperatures, and its growth is observed even at around $4{ }^{\circ} \mathrm{C}$ [37]. The presence of this bacteria strain may be responsible for severe problems with food storage.

The DSF assays were performed under no-growth conditions: without nutrients, only in an aqueous solution containing phosphate buffer $(0.3 \mathrm{M}, \mathrm{pH}=7.3)$. The samples were performed in the form of pastilles made of composite (Ag/PS/PVA) with the mass $76 \pm 8 \mathrm{mg}$ and such content of Ag@PS to achieve $1 \%$ of silver. The buffer solution was inoculated with $10^{5} \mathrm{CFU} \mathrm{ml} \mathrm{m}^{-1}$. The pastilles were placed in the solution, and afterwards, the mixture was shaken at $200 \mathrm{rpm}$ for $24 \mathrm{~h}$ at $37^{\circ} \mathrm{C}$. As a 
control sample, the pastill made of PS and PVA with a mass ratio 95:5 w/w was used. The bacterial concentration in the microbial suspension was determined by measuring optical density (O.D.) at wavelength $600 \mathrm{~nm}$. The percentage survival of bacterial cells in the system was calculated according to the following equation:

$$
S=\frac{C_{s}}{C_{c}} 100 \%
$$

where $C_{S}$-surviving cell concentration in the mixture with the composite sample after an appropriate time of incubation at $37^{\circ} \mathrm{C}, \mathrm{C}_{c}$-surviving cell concentration in the mixture with the control sample after the same period of incubation time as $C_{s}$.

The measurements of bacterial concentration were performed every hour for $6 \mathrm{~h}$, and the next measurement was performed after $24 \mathrm{~h}$.

Figure 6 displays the determined percentage survival $S \%$ of the studied bacterial strains vs. time of incubation in the presence of the pastilles made of Ag/PS/PVA composite containing 1\% of nanosilver and $5 \%$ of PVA. In the case of all studied systems after $24 \mathrm{~h}$ of incubation, there were not any survived cells. In a period of only $6 \mathrm{~h}$ number of bacterial cells decrease rapidly under the influence of the presence of the pastilles made of the nanocomposite. The survival of Escherichia coli after only $2 \mathrm{~h}$ of incubation with Ag/PS/PVA is $0.2 \%$. After a longer time of incubation, we did not observe any survived cells.
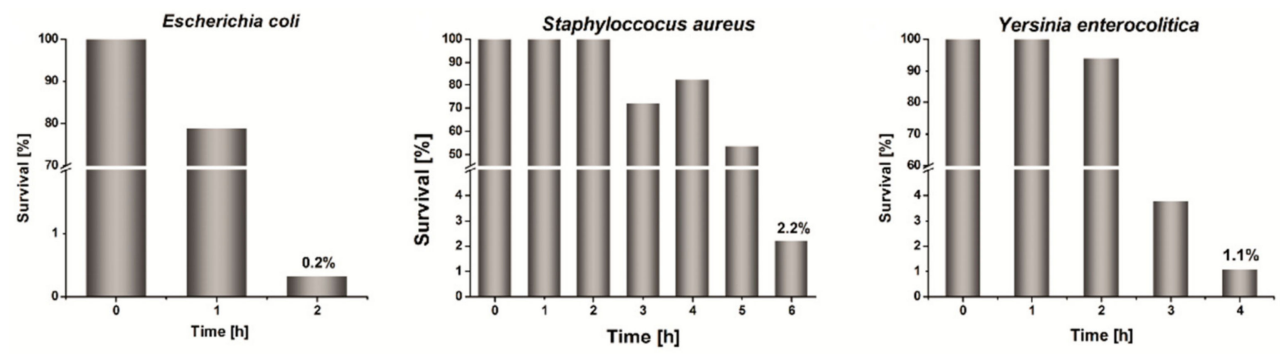

Figure 6. The bacterial survival under no bacteria growing conditions in DSF assay in the presence of pastilles made of Ag/PS/PVA (76 $\pm 8 \mathrm{mg}$ ) with silver content $1 \%$ and PVA content $5 \%$.

The survival of Staphylococcus aureus in the presence of the studied nanocomposite is higher and after $6 \mathrm{~h}$ is $2.2 \%$, but after $24 \mathrm{~h}$ of incubation, we did not observe survived cells. The observed differences in susceptibility between Escherichia coli and Staphylococcus aureus are a consequence of the different structure and the composition of their cell membranes. Staphylococcus aureus is a representative of Gram(+) bacteria; therefore, its cell membrane is significantly thicker, and permeation through the membrane is more complicated than in the case of Gram(-) bacteria.

The survival of Yersinia enterocolitica is higher than Escherichia coli and lower than Staphylococcus aureus. After $4 \mathrm{~h}$ of incubation, in the presence of Ag/PS/PVA, the survival is $1.1 \%$, whereas after $5 \mathrm{~h}$ the surviving cells were not observed.

Notably, the biocidal activity of the developed nanocomposites is significantly higher than for the analogical composites without the addition of PVA. The survival of Staphylococcus aureus after $24 \mathrm{~h}$ of incubation in the presence of pastille, made of Ag@PS and dispersed in PS with 4\% silver, is almost $90 \%$ [20].

Our results revealed that the doping of polystyrene nanocomposites with a hydrophilic polymer increases their bioactivity.

The performed antibacterial assays proved that the fabricated nanocomposites could be successfully used as biocidal materials against pathogenic bacteria, both $\operatorname{Gram}(-)$ and $\operatorname{Gram}(+)$.

\section{Conclusions}

In summary, we propose a new approach for the preparation of highly bioactive and thermoprocessable nanocomposites. In the prepared materials, polystyrene-grafted silver nanoparticles 
(Ag@PS) play the role of bioactive fulfilment, and a mixture of polystyrene (PS) and poly(vinyl alcohol) PVA is the polymer matrix.

We also report the method allowing us to fabricate narrowly sized dispersive (5.96 $\pm 1.02 \mathrm{~nm}$ ) silver nanoparticles (N-AgNPs) on a scale of hundreds of milligrams/several grams. Nitroxide radicals stabilize the synthesized nanoparticles, and thanks to this, they can be readily grafted with polymer chains via radical coupling during radical polymerization. Polystyrene-grafted silver nanoparticles (Ag@PS), prepared in this way, are perfectly dispersible in a mixture of PS with PVA (95:5 w/w). Such nanocomposites are thermally stable and, thus, can be thermally processed into pellets, pastilles, foils, plates, and other elements.

Kirby-Bauer (K-B) and Dynamic Shake Flask (DSF) assays showed high antibacterial activity against a series of $\operatorname{Gram}(-)$ and $\operatorname{Gram}(+)$ bacteria strains of the fabricated nanocomposites. Regarding the silver content, $2 \%$ of the biocidal activity of the nanocomposites is very high, and when the content is only $0.5 \%$, the activity can be evaluated as being effective.

The doping of Ag/PS composites with PVA gives antibacterial activity at a low concentration of nanosilver $(0.5 \%)$. Most likely, the presence of hydrophilic admixture in the nanocomposites enables water migration inside the material, which makes the releasing of silver nanoparticles and ions from the polymer matrix easier.

Due to the thermal processability and antibacterial activity at low silver content, the fabricated nanocomposites are highly promising materials for the fabrication of medical equipment such as, among others, surgical instruments, prosthesis, coatings, and dental tools.

Supplementary Materials: The following are available online at http://www.mdpi.com/2079-4991/10/11/2245/s1, Figure S1: The photograph of the lab set employed for the N-AgNPs preparation taken during the synthesis. Figure S2: The elugram obtained from SEC for PSF sample. Figure S3: The molecular weights distribution in the sample PSF determined on the base of SEC analyses.

Author Contributions: E.M.: conceptualization, methodology, writing-original draft preparation, editing; A.K.: investigations, measurements and syntheses performing. All authors have read and agreed to the published version of the manuscript.

Funding: This research was funded by the Polish Ministry of Science and Higher Education grant number 501-D407-56-0122777 (Inkubator+) and APC was funded by University of Warsaw.

Acknowledgments: Antibacterial activity assays have been performed by BACTrem-start-up company of the University of Warsaw, Faculty of Biology. We would like to thank OST-MED Sp. Z o. o. for all the support and cooperation during the studies. The authors thank Piotr Cieciórski, one of the members of our group, MSc, (University of Warsaw, Faculty of Chemistry, Warsaw, Poland) for preparing the Graphical Abstract.

Conflicts of Interest: The authors declare no conflict of interest.

\section{References}

1. Dallas, P.; Sharma, V.K.; Zboril, R. Silver polymeric nanocomposites as advanced antimicrobial agents: Classification, synthetic paths, applications, and perspectives. Adv. Colloid Interface Sci. 2011, 166, 119-135. [CrossRef] [PubMed]

2. Zhang, X.-F.; Liu, Z.-G.; Shen, W.; Gurunathan, S. Silver nanoparticles: Synthesis, characterization, properties, applications, and therapeutic approaches. Int. J. Mol. Sci. 2016, 17, 1534. [CrossRef] [PubMed]

3. Sriram, M.I.; Kalishwaralal, K.; Barathmanikanth, S.; Gurunathani, S. Size-based cytotoxicity of silver nanoparticles in bovine retinal endothelial cells. Nanosci. Methods 2012, 1, 56-77. [CrossRef]

4. Stoehr, L.C.; Gonzalez, E.; Stampfl, A.; Casals, E.; Duschl, A.; Puntes, V.; Oostingh, G.J. Shape matters: Effects of silver nanospheres and wires on human alveolar epithelial cells. Part. Fibre Toxicol. 2011, 8, 36. [CrossRef] [PubMed]

5. Suresh, A.K.; Pelletier, D.A.; Wang, W.; Morrell-Falvey, J.L.; Gu, B.; Doktycz, M.J. Cytotoxicity induced by engineered silver nanocrystallites is dependent on surface coatings and cell types. Langmuir 2012, 28, 2727-2735. [CrossRef]

6. Gupta, R.K.; Kennel, E.; Kim, K.-J. Polymer Nanocomposites Handbook; CRC Press: Boca Raton, FL, USA, 2009. 
7. Bourgeat-Lami, E. Hybrid Materials, Synthesis, Characterization and Applications; WIley-VCH Verlag GmbH\&Co, Wien: Weinheim, Germany, 2007.

8. Kumar, S.K.; Jouault, N.; Benicewicz, B.; Neely, T. Nanocomposites with Polymer Grafted Nanoparticles. Macromolecules 2013, 46, 3199-3214. [CrossRef]

9. Chen, J.; Fan, L.; Yang, C.; Wang, S.; Zhang, M.; Xu, J.; Luo, S. Facile synthesis of Ag nanoparticles-loaded chitosan antibacterial nanocomposite and its application in polypropylene. Int. J. Biol. Macromol. 2020, 161, 1286-1295. [CrossRef]

10. Iqbal, M.; Zafar, H.; Mahmood, A.; Niazi, M.B.K.; Aslam, M.W. Starch-Capped Silver Nanoparticles Impregnated into Propylamine-Substituted PVA Films with Improved Antibacterial and Mechanical Properties for Wound-Bandage Applications. Polymers 2020, 12, 2112. [CrossRef]

11. Salleh, A.; Naomi, R.; Utami, N.D.; Mohammad, A.W.; Mahmoudi, E.; Mustafa, N.; Fauzi, M.B. The potential of silver nanoparticles for antiviral and antibacterial applications: A mechanism of action. Nanomaterials 2020, 10, 1566. [CrossRef]

12. Adibelli, M.; Ozcelik, E.; Batibay, G.S.; Arasoglu, T.O.; Arsu, N. A facile and versatile route for preparation AgNp nanocomposite thin films via thiol-acrylate photopolymerization: Determination of antibacterial activity. Prog. Org. Coat. 2020, 143, 105620. [CrossRef]

13. Yang, Y.; Zhang, Z.; Wan, M.; Wang, Z.; Zou, X.; Zhao, Y.; Sun, L. A Facile Method for the Fabrication of Silver Nanoparticles Surface Decorated Polyvinyl Alcohol Electrospun Nanofibers and Controllable Antibacterial Activities. Polymers 2020, 12, 2486. [CrossRef] [PubMed]

14. Wünsch, J.R. Polystyrene: Synthesis, Production and Applications; iSmithers Rapra Publishing: Shawbury, UK, 2000; Volume 112.

15. Cieplak, P.; Megiel, E.; Kaim, A. Penultimate unit effects in the free-radical copolymerization of styrene with acrylonitrile according to theoretical thermochemistry. J. Polym. Sci. Part A: Polym. Chem. 2002, 40, 3592-3603. [CrossRef]

16. Modjarrad, K.; Ebnesajjad, S. Handbook of Polymer Applications in Medicine and Medical Devices; Elsevier: Amsterdam, The Netherlands, 2013.

17. Vodnik, V.; Božanić, D.K.; Džunuzović, J.V.; Vukoje, I.; Nedeljković, J. Silver/polystyrene nanocomposites: Optical and thermal properties. Polym. Compos. 2012, 33, 782-788. [CrossRef]

18. Kai, Z.; Qiang, F.; Jinghui, F.; Dehui, Z. Preparation of Ag/PS composite particles by dispersion polymerization under ultrasonic irradiation. Mater. Lett. 2005, 59, 3682-3686. [CrossRef]

19. Wang, D.; An, J.; Luo, Q.; Li, X.; Li, M. A convenient approach to synthesize stable silver nanoparticles and silver/polystyrene nanocomposite particles. J. Appl. Polym. Sci. 2008, 110, 3038-3046. [CrossRef]

20. Krystosiak, P.; Tomaszewski, W.; Megiel, E. High-density polystyrene-grafted silver nanoparticles and their use in the preparation of nanocomposites with antibacterial properties. J. Colloid Interface Sci. 2017, 498, 9-21. [CrossRef] [PubMed]

21. Rusu, E.; Airinei, A.; Barboiu, V.; Timpu, D. Some characteristics of poly (vinyl alcohol) with azido aromatic groups. J. Optoelectron. Adv. Mater. 2007, 9, 1044.

22. Teodorescu, M.; Bercea, M.; Morariu, S. Biomaterials of PVA and PVP in medical and pharmaceutical applications: Perspectives and challenges. Biotechnol. Adv. 2019, 37, 109-131. [CrossRef]

23. Abdallah, O.M.; EL-Baghdady, K.Z.; Khalil, M.M.; El Borhamy, M.I.; Meligi, G.A. Antibacterial, antibiofilm and cytotoxic activities of biogenic polyvinyl alcohol-silver and chitosan-silver nanocomposites. J. Polym. Res. 2020, 27, 1-9. [CrossRef]

24. Kaim, A.; Szydłowska, J.; Piotrowski, P.; Megiel, E. One-pot synthesis of gold nanoparticles densely coated with nitroxide spins. Polyhedron 2012, 46, 119-123. [CrossRef]

25. EUCAST Disk Diffusion Method for Antimicrobial Susceptibility Testing-Version 5.0 (1 January 2015). Available online: www.eucast.org (accessed on 1 January 2015).

26. Swiech, O.; Bilewicz, R.; Megiel, E. TEMPO coated Au nanoparticles: Synthesis and tethering to gold surfaces. RSC Adv. 2013, 3, 5979. [CrossRef]

27. Swiech, O.; Hrynkiewicz-Sudnik, N.; Palys, B.; Kaim, A.; Bilewicz, R. Gold Nanoparticles Tethered to Gold Surfaces Using Nitroxyl Radicals. J. Phys. Chem. C 2011, 115, 7347-7354. [CrossRef]

28. Gozdziewska, M.; Cichowicz, G.; Markowska, K.; Zawada, K.; Megiel, E. Nitroxide-coated silver nanoparticles: Synthesis, surface physicochemistry and antibacterial activity. RSC Adv. 2015, 5, 58403-58415. [CrossRef] 
29. Zawada, K.; Tomaszewski, W.; Megiel, E. A smart synthesis of gold/polystyrene core-shell nanohybrids using TEMPO coated nanoparticles. RSC Adv. 2014, 4, 23876-23885. [CrossRef]

30. Underwood, S.; Mulvaney, P. Effect of the solution refractive index on the color of gold colloids. Langmuir 1994, 10, 3427-3430. [CrossRef]

31. Vodnik, V.V.; Vuković, J.V.; Nedeljković, J.M. Synthesis and characterization of silver-Poly(methylmethacrylate) nanocomposites. Colloid. Polym. Sci. 2009, 287, 847-851. [CrossRef]

32. Nicolas, J.; Guillaneuf, Y.; Lefay, C.; Bertin, D.; Gigmes, D.; Charleux, B. Nitroxide-mediated polymerization. Prog. Polym. Sci. 2013, 38, 63-235. [CrossRef]

33. Megiel, E. Surface modification using TEMPO and its derivatives. Adv. Colloid Interface Sci. 2017, 250, 158-184. [CrossRef]

34. Lien, E.J.; Hansch, C.; Anderson, S.M. Structure-activity correlations for antibacterial agents on gram-positive and gram-negative cells. J. Med. Chem. 1968, 11, 430-441. [CrossRef]

35. Silvan, J.M.; Zorraquin-Peña, I.; Gonzalez de Llano, D.; Moreno-Arribas, M.; Martinez-Rodriguez, A.J. Antibacterial activity of glutathione-stabilized silver nanoparticles against Campylobacter multidrug-resistant strains. Front. Microbiol. 2018, 9, 458. [CrossRef]

36. Pepe, J.C.; Miller, V.L. Yersinia enterocolitica invasin: A primary role in the initiation of infection. Proc. Natl. Acad. Sci. USA 1993, 90, 6473-6477. [CrossRef]

37. Li, C.; Murugaiyan, J.; Thomas, C.; Alter, T.; Riedel, C. Isolate Specific Cold Response of Yersinia enterocolitica in Transcriptional, Proteomic, and Membrane Physiological Changes. Front. Microbiol. 2020, 10, 3037. [CrossRef]

Publisher's Note: MDPI stays neutral with regard to jurisdictional claims in published maps and institutional affiliations.

(C) 2020 by the authors. Licensee MDPI, Basel, Switzerland. This article is an open access article distributed under the terms and conditions of the Creative Commons Attribution (CC BY) license (http://creativecommons.org/licenses/by/4.0/). 